Original article

\title{
Smoking cessation treatment outcomes among people with and without mental and substance use disorders: An observational real-world study
}

\author{
João Mauricio Castaldelli-Maia ${ }^{\mathrm{a}, \mathrm{b}, \mathrm{c}, \mathrm{d}, *}$, Aline Rodrigues Loreto ${ }^{\mathrm{a}}$, \\ Bruna Beatriz Sales Guimarães-Pereira ${ }^{a}$, Carlos Felipe Cavalcanti Carvalhob ${ }^{\mathrm{b}}$, Felipe Gil ${ }^{\mathrm{c}}$, \\ Fernanda Piotto Frallonardo ${ }^{c}$, Flávia Ismael ${ }^{c}$, Arthur Guerra de Andrade $^{a, c, d}$, \\ Antonio Ventriglio ${ }^{\mathrm{e}}$, Kimber P. Richter ${ }^{\mathrm{f}}$, Dinesh Bhugrag
}

a Department of Neuroscience, Medical School, ABC Foundation, André Santo, SP, Brazil

b Clima Clinic, São Paulo, SP, Brazil

${ }^{\mathrm{c}}$ ABC Center for Mental Health Studies, Santo André, SP, Brazil

d Department of Psychiatry, Medical School, University of São Paulo, São Paulo, SP, Brazil

e Department of Clinical and Experimental Medicine, University of Foggia, Foggia, Italy

${ }^{\mathrm{f}}$ Department of Preventive Medicine and Public Health, University of Kansas School of Medicine, Kansas City, KS, USA

${ }^{g}$ Health Service \& Population Research Department, Institute of Psychiatry, King's College, London, UK

\section{A R T I C L E I N F O}

\section{Article history:}

Received 12 July 2017

Received in revised form 11 February 2018

Accepted 28 February 2018

Available online 30 March 2018

\section{Keywords:}

Smoking

Tobacco

Treatment

Mental health

Substance use disorders

\begin{abstract}
A B S T R A C T
Background: There is a lack of studies evaluating smoking cessation treatment protocols which include people with and without mental and substance use disorders (MSUD), and which allows for individuals with MSUD undergoing their psychiatric treatment.

Methods: We compared treatment success between participants with $(n=277)$ and without $(n=419)$ MSUD among patients in a 6-week treatment provided by a Brazilian Psychosocial Care Center (CAPS) from 2007 to 2013. Sociodemographic, medical and tobacco use characteristics were assessed at baseline. Tobacco treatment consisted of 1) group cognitive behavior therapy, which included people with and without MSUD in the same groups, and 2) pharmacotherapy, which could include either nicotine patches, nicotine gum, bupropion or nortriptyline. For participants with MSUD, tobacco treatment was integrated into their ongoing mental health treatment. The main outcome was 30-day point prevalence abstinence, measured at last day of treatment.

Results: Abstinence rates did not differ significantly between participants with and without MSUD (31.1\% and $34.4 \%$, respectively). Variables that were significantly associated with treatment success included years smoking, the Heaviness of Smoking Index, and use of nicotine patch or bupropion.

Conclusion: The inclusion of individuals with and without MSUD in the same protocol, allowing for individuals with MSUD undergoing their psychiatric treatment, generates at least comparable success rates between the groups. Predictors of treatment success were similar to those found in the general population. Facilities that treat patients with MSUD should treat tobacco use in order to reduce the disparities in morbidity and mortality experienced by this population.
\end{abstract}

(C) 2018 Elsevier Masson SAS. All rights reserved.

\section{Introduction}

Cigarette smoking is the primary preventable cause of death in the western world [1]. Among patients with mental disorders,

\footnotetext{
* Corresponding author at: Disciplinas de Psiquiatria e Psicologia Médica, Clima Clinic, Rua Guarará 529, Cj. 127, 01425-001, Jardins, São Paulo, SP, Brazil.

E-mail address: jmcmaia@usp.br (J.M. Castaldelli-Maia).
}

tobacco use prevalence is two to four times higher than that found in the general population [2,3]. Numerous biological, psychological and social factors may explain these high rates of smoking and include lack of smoking cessation treatment in mental health programs [5] as well as the use of tobacco for self-medication of psychiatric symptoms [4].

These high rates of smoking contribute to extremely high rates of morbidity and mortality [4]. People with severe mental illness die 25 years younger than the general population, and most of this 
premature mortality is due to tobacco use [6]. For example, people with severe depression, alcohol-related disorders and schizophrenia have high mortality rates due to vascular disease and cancer [4]. Smoking also complicates treatment of some mental disorders by reducing blood levels of neuroleptics, such as olanzapine, clozapine, haloperidol, chlorpromazine, and fluphenazine. Therefore, smokers may require higher doses to achieve a therapeutic effect, and may be at increased risk of side effects from their psychotherapeutic medications [7].

Progress in the development of tailored cessation interventions for people with mental and substance use disorders (MSUD) has been slow, in part because most smokers with mental disorders are still excluded from most smoking cessation trials [7-10]. A growing body of literature, however, demonstrates that tobacco treatment does help smokers with mental disorders quit, and that neither treatment of tobacco dependence nor quitting smoking have adverse effects on psychiatric symptoms or serious adverse events such as death or hospitalization [11-13]. Gold-standard treatment of tobacco dependence for people with MSUD should combine cognitive-behavioral therapies with motivational enhancement interventions and integrate tobacco cessation pharmacotherapy into ongoing psychiatric care [10].

A number of studies have shown that, among individuals with MSUD, tobacco treatment is effective [4,15,17], and that retention rates are very good when patients are treated for tobacco dependence while they are undergoing psychiatric treatment [3]. Unfortunately, patients with MSUD who receive treatment for their tobacco use appear to achieve lower cessation rates than smokers in the general population. Few existing trials were designed to directly compare the success rates of smokers with and without MSUD. A recent large-scale randomized controlled trial [2] that was designed to make this comparison found that treatment with either bupropion or NRT resulted in significantly lower quit rates for individuals with MSUD when compared to those without MSUD. At week 12, 20\% participants with MSUD who received medication were abstinent, compared to $26 \%$ of those without MSUD who received medication.

Most of these studies, however, were clinical trials implemented in ideal conditions, which may not accurately reflect achievable outcomes in real-world clinical practice. Brazilian Psychosocial Care Centers (CAPS) are healthcare units that promote comprehensive care for people with severe and persistent mental disorders. The goal of CAPS is to enable patients to live in society, work, study and enjoy their full rights as citizens [14]. CAPS is an ideal setting for treating tobacco dependence among those with mental disorders [15]. CAPS employs multidisciplinary teams, composed of professionals specializing in mental health or addiction treatment [16], and can obtain materials and medications for smoking cessation treatment from the Brazilian National Cancer Institute (INCA).

Within CAPS, as in programs in other countries, perceptions that smokers with mental disorders do not want to quit, or are unable to quit, are major barriers to integrating tobacco treatment into mental health treatment [18-20]. Hence, understanding the impact of treatment in a real world clinical setting, and the relative effectiveness of treatment among people with and without comorbid mental illness, should shed light on the extent to which the culture of MSUD centers affects cessation outcomes and may provide clues regarding how existing barriers might be overcome.

The purpose of this study was to compare 30-day point abstinence prevalence at the end of a 6-week tobacco treatment program. taking into account important covariates (i.e., sociodemographic, smoking characteristics, type of treatment) that are known to influence the success of treatment. All participants received smoking cessation treatment in a Brazilian CAPS unit, by the health care professionals that staffed that unit. This permits a direct comparison of cessation rates across individuals with and without MSUD, who received care together in a real-world treatment center.

\section{Methods}

\subsection{Ethics approval}

This study was approved by the Faculty of Medicine of the ABC Foundation Ethics Committee.

\subsection{Design of the study}

\subsubsection{Study overview}

The study used a pretest, posttest observational design conducted as part of real-world clinical practice. It reports the outcomes of a 6-week treatment protocol that was adopted in April 2007 by a CAPS Alcohol and Drug (AD) unit located in São Caetano do Sul (CAPS-AD-SCS) in the state of São Paulo, Brazil. This protocol included medical consultations, group psychotherapy, and systematic data collection at each patient visit. Patient data was accessed through retrospective analysis of unit medical records. The main outcome measure was defined as 30-day continuous abstinence assessed at the last day of the treatment, named 30-day point prevalence abstinence. Patients could participate in the treatment protocol more than one time, if they relapsed. However, for the purpose of this study, only the first treatment episode and resulting outcomes for each patient were included in analyses.

\subsubsection{Medical/psychiatry consultations}

A psychiatry resident supervised by a fully qualified psychiatrist typically conducted consultations. Consultations included assessing withdrawal, determining need for pharmacotherapy, monitoring side effects of pharmacotherapy, and evaluating any need for dosing adjustments. Medication options included bupropion, nortriptyline, and nicotine replacement therapy such as patch or gum. Varenicline was not included in the protocol as it was not available through the National Tobacco Program. Considering that this was a real-life (naturalistic) study, criteria for allocation for pharmacologic treatment were guided by availability, clinical team agreement, patient preferences, and previous use. Patients could receive more than one tobacco cessation medication. Physicians had the ability to refer patients to other specialist physicians or psychiatrists as needed, during or after the 6-week treatment. Physicians did not participate in any of the group psychotherapy sessions, and no data from the individual sessions were shared with CAPS-AD unit staff or other patients in the psychotherapy group.

\subsubsection{Group psychotherapy}

The total duration of the treatment protocol was 6 weeks. During this period, each patient had six scheduled sessions of group therapy and four scheduled individual visits with a psychiatrist $(\mathrm{t} 1=0, \mathrm{t} 2=1$ week, $\mathrm{t} 3=3$ weeks, and $\mathrm{t} 4=6$ weeks $)$.

The psychotherapy groups were based on the principles of cognitive-behavioral psychotherapy and included individuals with and without MSUD. The groups included up to 15 persons for each 6 -week treatment. The main topics for discussion in group therapy sessions were the dangers of smoking, craving, coping tactics and recognition of withdrawal symptoms, the difficulties and benefits of treatment, and relapse prevention.

\subsection{Sample}

The sample consisted of all patients who participated in the CAPS-AD-SCS Smoking Cessation Treatment protocol between the 
years of 2007 to 2013. The sample included people without MSUD even though the treatment setting was a psychosocial care center. This occurred because the clinic adopted the national treatment protocol and actively solicited referrals from primary care and other medical/social service settings that served people without MSUD in the city.

\subsubsection{Inclusion/exclusion criteria}

At the beginning of treatment of in CAPS-AD-SCS, all patients filled out an initial questionnaire containing sociodemographic information, medical information, and tobacco use history.

Inclusion criteria included patients who had been seen by any member of the multidisciplinary team of CAPS-AD-SCS at least once for the Smoking Cessation Treatment protocol. In addition, participants had to agree to participate in the Smoking Cessation Treatment protocol. Exclusion criteria included living outside the city of São Caetano do Sul; being younger than 18 or older than 65; did not legibly complete the initial questionnaire; being pregnant. Last, smokers with a history of substance use disorder had to be abstinent for a minimum period of one year to be included in the present study - this unit has adopted this requirement based on data suggesting that delayed treatment for tobacco use yields the best outcomes for substance use disorders and tobacco dependence $[3,15,21]$.

\subsection{Variables}

\subsubsection{MSUD}

This variable-mental and/or substance use disorder (MSUD)was defined based on the medical record item that assessed whether the patient had been diagnosed with an MSUD in the last year. Both patients with and without MSUD were recruited and treated for tobacco dependence in the same way.

\subsubsection{Covariates}

Sociodemographic variables included gender, age, education, and family income. Medical variables included any comorbidity and participation in physical activity. Tobacco use history included duration of smoking (in years), cigarettes per day, time to first cigarette, difficulty not smoking in areas where it is prohibited, the most difficult cigarette to abstain from, smoking while sick, and the Heaviness of Smoking Index (HSI) [22], which is composed of time to first cigarette and cigarettes smoked per day. Type of cessation pharmacotherapy received was also assessed (nicotine patch, nicotine gum, bupropion, and nortriptyline).

\subsection{Outcome}

The outcome measure (treatment success) was defined as 30day point prevalence abstinence - the person self-reported abstinence for at least 30 consecutively days prior to the last day of the 6-week treatment. Several studies use the 30-day abstinence measure to evaluate the smoking treatment outcome [23-26]. In addition, as a secondary measure of outcome we calculated treatment adherence by presence of the individual in all medical consultation and group psychotherapy sessions.

\subsection{Statistical analysis}

Our main outcome was evaluated via an intent-to-treatanalysis in which all patients who enrolled in the treatment protocol, regardless of treatment adherence, were included in analyses. Participants lost to follow up were counted as smokers. Age and cigarettes per day variables were treated as ordinal variables in analyses, which were performed using STATA version
11.2 (Stata Corp, Texas, U.S., 2009). First, we used Chi-square analyses to determine covariate variables between people with and without MSUD; our cutoff for statistical significance was set at $\mathrm{p}<.05$. Second, we ran univariate logistic regression models for the outcome of interest (30-day point prevalence abstinence) for MSUD status and all covariates. Third, we ran a linear correlation model including MSUD status and the covariates. We selected the variables to include in the final logistic regression multivariate model based on the statistical significance in the univariate regression $(\mathrm{p}<.10$ for hypertension, HSI, time of smoking, nicotine patch) and the findings of previous studies. Time to first cigarette and cigarettes per day were highly correlated with HIS and were not included in the final model. Age was highly correlated with duration of smoking and was also not included.

\section{Results}

Table 1 presents the descriptive analysis of the sample. MSUD status was significantly associated with male gender (45.1\% versus $29.1 \%)$, any health care ( $76.1 \%$ versus $57.5 \%)$, medication use $(79.1 \%$ versus $60.0 \%$ ), 31 years or more of smoking (58.1\% versus $52.5 \%$ ), and smoking 21 cigarettes or more per day (55.2\% versus $44.4 \%$ ). Patients without MSUD were significantly more likely to use bupropion (54.8 versus 34.3\%). Patients with MSUD scored markedly, but not significantly higher on the HSI than those without ( $45.8 \%$ versus $36.5 \%$, respectively, were categorized as highly dependent). Fig. 1 presents rates for adherence and 30-day point prevalence cessation for MSUD and non-MSUD smokers. No significant difference was found.

Table 2 presents the results of the univariate regression models for 30-day point prevalence abstinence (treatment success). There was no overall difference between the MSUD and non-MSUD group. However, age $(\mathrm{OR}=1.56,95 \% \mathrm{CI}=1.19-2.05, \mathrm{p}=0.001)$, years smoking $(\mathrm{OR}=1.28,95 \% \mathrm{CI}=1.09-1.51, \mathrm{p}=0.002)$, and nicotine patch use $(\mathrm{OR}=2.76,95 \% \mathrm{CI}=1.05-7.28, \mathrm{p}=0.039)$ were significantly associated with treatment success. Conversely, cigarettes per day $(\mathrm{OR}=0.80,95 \% \mathrm{CI}=0.68-0.95, \mathrm{p}=0.015)$ and Heaviness of Smoking Index $(\mathrm{OR}=0.75,95 \% \mathrm{CI}=0.64-0.87, \mathrm{p}<0.001)$ were associated with treatment failure (not achieving 30-day abstinence). There was a marked but non-significant association between systemic arterial hypertension and treatment success $(\mathrm{OR}=1.35,95 \% \mathrm{CI}=0.951 .91, \mathrm{p}=0.087)$.

Table 3 presents linear correlations between MSUD status and the covariates. There were strong correlations between: HSI and cigarettes per day (0.79); HSI and time to first cigarette (0.76); time of smoking and age (0.55); cigarettes per day and time to first cigarette (0.32). Importantly, HSI is derived from time to first cigarette smoking and cigarettes per day. Thus, from these three covariates we opted to include only HSI in the multivariate model. Considering that duration of years smoking was significantly different between MSUD and non-MSUD groups, and was significantly associated with treatment success in the univariate regression model, we decided to include it and not age in the multivariate model. There were no significant direct correlations between MSUD status and any of the covariates.

Table 4 presents the results of the multivariate logistic regression model for 30-day point prevalence abstinence (treatment success). There was no significant difference between the MSUD and non-MSUD group ( $\mathrm{aOR}=0.98,95 \% \mathrm{CI}=0.69-1.39$, $\mathrm{p}=0.945)$. However, years smoking $(\mathrm{aOR}=1.33,95 \% \mathrm{CI}=1.13-$ $1.58, \mathrm{p}=0.001)$, nicotine patch use $(\mathrm{aOR}=2.96,95 \% \mathrm{CI}=1.10-7.95$, $\mathrm{p}=0.031)$, and bupropion use $(\mathrm{aOR}=1.45,95 \mathrm{Cl} \%=1.03-2.03$, $\mathrm{p}=0.030$ ) were associated with treatment success. Conversely, HSI was associated with treatment failure $(\mathrm{aOR}=0.71,95 \mathrm{CI}$ $\%=0.61-0.84, \mathrm{p}<0.001)$. 
Table 1

Sociodemographic, medical and smoking profile descriptive analysis of the sample ( $\mathrm{n}=696)$ of smokers treated at CAPS-AD São Caetano do Sul, $2007-2013$.

\begin{tabular}{|c|c|c|c|c|c|c|}
\hline & \multicolumn{2}{|c|}{$\begin{array}{l}\text { MSUD } \\
(n=277)\end{array}$} & \multicolumn{2}{|c|}{$\begin{array}{l}\text { non-MSUD } \\
(\mathrm{n}=419)\end{array}$} & \multirow[t]{2}{*}{ Chi } & \multirow[t]{2}{*}{$p$} \\
\hline & $n$ & $\%$ & $n$ & $\%$ & & \\
\hline \multicolumn{7}{|l|}{ Sociodemographic } \\
\hline \multicolumn{7}{|l|}{ Gender } \\
\hline Female & 152 & 54.8 & 297 & 70.8 & \multirow[t]{2}{*}{18.6} & \multirow[t]{2}{*}{$<0.001$} \\
\hline Male & 125 & 45.1 & 122 & 29.1 & & \\
\hline \multicolumn{7}{|l|}{ Education } \\
\hline None or Elementary (partial) & 84 & 30.3 & 93 & 22.3 & \multirow[t]{6}{*}{8.7} & \multirow[t]{6}{*}{0.121} \\
\hline Elementary (complete) & 29 & 10.4 & 38 & 9.0 & & \\
\hline High-school (partial) & 36 & 13.0 & 59 & 14.1 & & \\
\hline High-school (complete) & 59 & 21.3 & 115 & 27.5 & & \\
\hline Undergraduate (partial) & 41 & 14.8 & 58 & 13.8 & & \\
\hline Undegraduate (complete) or Graduate & 28 & 10.1 & 55 & 13.2 & & \\
\hline \multicolumn{7}{|l|}{ Family Income } \\
\hline Up to 1 minimum salary & 61 & 22.8 & 75 & 18.3 & \multirow[t]{3}{*}{2.3} & \multirow[t]{3}{*}{0.305} \\
\hline From 2 to 3 minimum salaries & 127 & 47.4 & 191 & 46.7 & & \\
\hline 4 minimum salaries or more & 89 & 32.1 & 153 & 36.5 & & \\
\hline Age Group & & & & & & \\
\hline Young Adult (20-39 years old) & 38 & 13.8 & 61 & 14.6 & 0.7 & 0.705 \\
\hline Adult ( $40-59$ years old) & 172 & 62.3 & 269 & 64.2 & & \\
\hline Older Adult ( $>60$ years old) & 66 & 23.9 & 89 & 21.2 & & \\
\hline Medical Profile & & & & & & \\
\hline Any Comorbidities & & & & & & \\
\hline & 89 & 32.1 & 150 & 35.8 & 1.0 & 0.318 \\
\hline Systemic Arterial Hypertension (SAH) & & & & & & \\
\hline & 70 & 25.3 & 125 & 29.8 & 1.7 & 0.190 \\
\hline Any Health Care & & & & & & \\
\hline & 210 & 76.1 & 241 & 57.5 & 25.2 & $<0.001$ \\
\hline Any Medication Regulary Use & & & & & & \\
\hline & 216 & 79.1 & 250 & 60.0 & 27.7 & $<0.001$ \\
\hline Regular Physical Activity & & & & & & \\
\hline & 103 & 37.3 & 174 & 41.8 & 1.4 & 0.236 \\
\hline Smoking Profile & & & & & & \\
\hline Years smoking & & & & & & \\
\hline Less than 21 years & 59 & 21.3 & 66 & 15.8 & 11.7 & 0.008 \\
\hline From 21 to 30 years & 57 & 20.6 & 133 & 31.7 & & \\
\hline From 31 to 40 years & 99 & 35.7 & 141 & 33.7 & & \\
\hline More than 40 years & 62 & 22.4 & 79 & 18.8 & & \\
\hline Cigarettes per day & & & & & & \\
\hline Less than 11 cigarettes & 20 & 7.2 & 37 & 8.8 & 10.5 & 0.015 \\
\hline From 11 to 20 cigarettes & 104 & 37.6 & 196 & 46.8 & & \\
\hline From 21 to 30 cigarettes & 69 & 24.9 & 100 & 23.9 & & \\
\hline Greater than 30 cigarettes & 84 & 30.3 & 86 & 20.5 & & \\
\hline Time to first cigarette & & & & & & \\
\hline Less than $5 \mathrm{~min}$ & 178 & 64.5 & 237 & 57.1 & 4.1 & 0.254 \\
\hline From 6 to $30 \mathrm{~min}$ & 71 & 25.7 & 133 & 32.0 & & \\
\hline From 30 to $60 \mathrm{~min}$ & 18 & 6.5 & 28 & 6.8 & & \\
\hline After $60 \mathrm{~min}$ & 9 & 3.3 & 17 & 4.1 & & \\
\hline Difficulty not smoking in areas where $i$ & & & & & & \\
\hline & 166 & 60.4 & 231 & 55.4 & 1.7 & 0.196 \\
\hline The most difficult cigarette to give up & & & & & & \\
\hline The first in the morning & 183 & 67.5 & 272 & 66.2 & 0.1 & 0.715 \\
\hline Smoking when being sick & & & & & & \\
\hline & 174 & 64.7 & 267 & 65.4 & 0.0 & 0.840 \\
\hline Heaviness of Smoking Index (HSI) & & & & & & \\
\hline Very Low Dependence & 31 & 11.2 & 57 & 13.6 & 6.8 & 0.078 \\
\hline Low to Moderate Dependence & 47 & 17.0 & 93 & 22.2 & & \\
\hline Moderate Dependence & 72 & 26.0 & 116 & 27.7 & & \\
\hline High Dependence & 127 & 45.8 & 153 & 36.5 & & \\
\hline Pharmacological Treatment & & & & & & \\
\hline Nicotine patch & 267 & 96.3 & 397 & 94.7 & 1.0 & 0.312 \\
\hline Nicotine gum & 45 & 16.2 & 59 & 14.0 & 0.6 & 0.433 \\
\hline Bupropion & 95 & 34.3 & 230 & 54.8 & 28.4 & 0.000 \\
\hline Nortriptyline & 10 & 3.6 & 19 & 4.53 & 0.3 & 0.550 \\
\hline
\end{tabular}

\section{Discussion}

The present study aimed to provide additional data from a realworld scenario to compare quit success rates in individuals with and without MSUD being treated in the same protocol, taking into account sociodemographics, smoking patterns, and treatment covariates, all of which could influence the success of treatment within this population. Patients with MSUD who received tobacco treatment integrated into their mental health treatment achieved cessation rates comparable to patients without these disorders. Nearly one in three were able to quit after a single round of treatment. The present study was important because few studies have evaluated smoking cessation treatment among MSUD patients $[4,15,17]$. Interestingly, the variables that were 
60
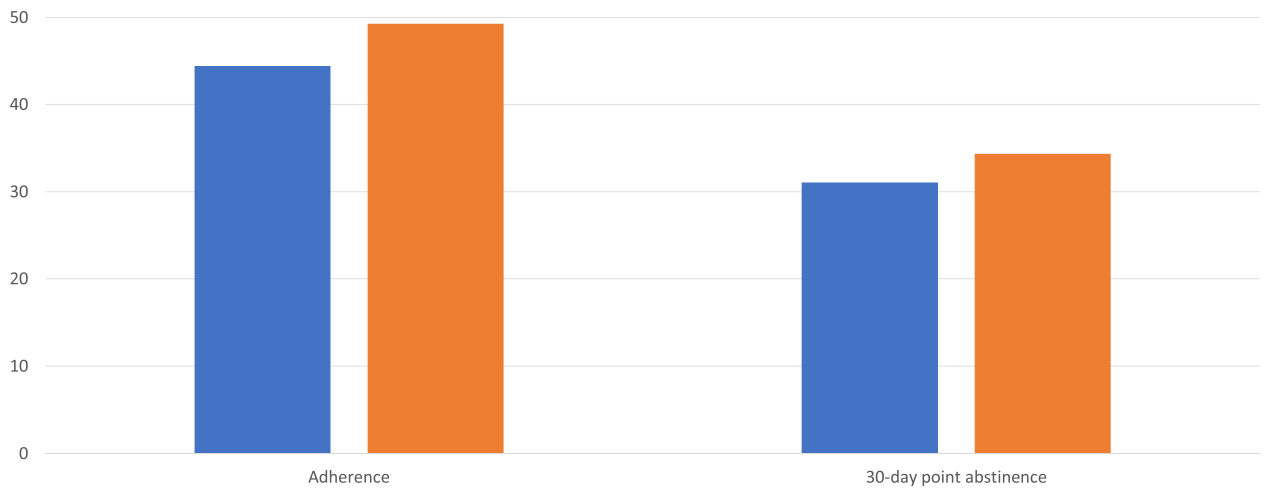

mSSUD $=$ non-MSUD

30-day point abstinence

Fig. 1. Adherence and 30-day point abstinence among smokers treated at CAPS-AD São Caetano do Sul, 2007-2013.

Table 2

Univariate regression models for 30-day abstinence among of smokers ( $n=696)$ who searched for treatment in CAPS-AD São Caetano do Sul. $2007-2013$.

\begin{tabular}{|c|c|c|c|c|c|c|}
\hline & OR & $\mathrm{z}$ & $95 \% \mathrm{CI}$ & Log likelihood & Pseudo R2 & $\mathrm{p}$ \\
\hline MSUD versus non-MSUD & 0.85 & -0.91 & $0.62-1.18$ & -441.2 & 0.0009 & 0.362 \\
\hline Gender & 0.82 & -1.11 & $0.59-1.15$ & -441.0 & 0.0014 & 0.265 \\
\hline Education & 0.95 & -0.89 & $0.87-1.05$ & -440.1 & 0.0009 & 0.303 \\
\hline Family Income & 1.07 & 0.67 & $0.86-1.34$ & -441.4 & 0.0005 & 0.898 \\
\hline Age Group & 1.56 & 3.27 & $1.19-2.05$ & -435.7 & 0.0124 & 0.001 \\
\hline Any Comorbidities & 1.12 & 0.68 & $0.80-1.56$ & -441.4 & 0.0005 & 0.495 \\
\hline Systemic Arterial Hypertension & 1.35 & 1.71 & $0.95-1.91$ & -440.2 & 0.0033 & 0.087 \\
\hline Any Health Care & 1.1 & 0.57 & $0.79-1.53$ & -440.4 & 0.0004 & 0.566 \\
\hline Medication Use & 1.17 & 0.92 & $0.83-1.65$ & -438.1 & 0.0010 & 0.357 \\
\hline Practice of physical activity & 1.11 & 0.65 & $0.80-1.53$ & -439.8 & 0.0005 & 0.517 \\
\hline Time of smoking & 1.28 & 3.1 & $1.09-1.51$ & -436.7 & 0.0111 & 0.002 \\
\hline Cigarettes per day & 0.8 & -2.43 & $0.68-0.95$ & -438.6 & 0.0068 & 0.015 \\
\hline Time to first cigarette & 0.69 & -3.69 & $0.57-0.84$ & -434.8 & 0.0154 & $<0.001$ \\
\hline Non-smoking places & 0.91 & -0.53 & $0.66-1.26$ & -437.8 & 0.0003 & 0.597 \\
\hline The most difficult cigarette & 0.89 & -0.62 & $0.63-1.26$ & -431.5 & 0.0004 & 0.538 \\
\hline Smoking while is sick & 0.79 & -1.36 & $0.56-1.10$ & -428.8 & 0.0021 & 0.175 \\
\hline Heaviness of Smoking Index & 0.75 & -3.73 & $0.64-0.87$ & -434.6 & 0.0158 & $<0.001$ \\
\hline Nicotine patch & 2.76 & 2.06 & $1.05-7.28$ & -439.0 & 0.0059 & 0.039 \\
\hline Nicotine gum & 1.08 & 0.37 & $0.70-1.68$ & -441.5 & 0.0002 & 0.712 \\
\hline Bupropion & 1.28 & 1.55 & $0.93-1.76$ & -440.4 & 0.0027 & 0.121 \\
\hline Nortriptyline & 1.25 & 0.57 & $0.58-2.69$ & -441.5 & 0.0004 & 0.569 \\
\hline
\end{tabular}

Table 3

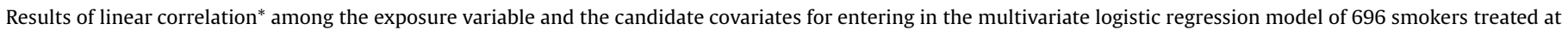
CAPS-AD SCS, 2007-2013.

\begin{tabular}{|c|c|c|c|c|c|c|c|c|c|c|}
\hline & MSUD & AG & YS & SHA & CPD & TTFC & HSI & Patch & Bup & G \\
\hline Mental/Substance Use Disorder (MSUD) & 1 & & & & & & & & & \\
\hline Age group (AG) & 0.02 & 1 & & & & & & & & \\
\hline Years smoking (YS) & 0.01 & 0.55 & 1 & & & & & & & \\
\hline Systemic Arterial Hypertension (SAH) & -0.04 & 0.22 & 0.18 & 1 & & & & & & \\
\hline Cigarettes per day (CPD) & 0.11 & -0.04 & 0.04 & 0.01 & 1 & & & & & \\
\hline Time to the first cigarette (TTFC) & 0.06 & -0.04 & 0.08 & -0.01 & 0.32 & 1 & & & & \\
\hline Heaviness of smoking index (HSI) & 0.08 & -0.05 & 0.07 & 0.01 & 0.79 & 0.76 & 1 & & & \\
\hline Nicotine patch (Patch) & 0.03 & -0.02 & -0.02 & 0.01 & 0.01 & 0.06 & 0.02 & 1 & & \\
\hline Buproprion (Bup) & -0.20 & -0.09 & -0.06 & -0.10 & 0.08 & 0.03 & 0.08 & 0.02 & 1 & \\
\hline Gender (G) & 0.016 & -0.01 & 0.04 & -0.01 & 0.15 & 0.02 & 0.11 & -0.06 & -0.01 & 1 \\
\hline
\end{tabular}

significantly associated with 30-day smoking abstinence (years smoking, HSI, nicotine patch and bupropion) were those also found to be important for smoking cessation treatment in the general population $[8,9]$.

Some recent studies support the idea that patients with psychiatric disorders should receive the same treatment for nicotine dependence that is given to the general population as long as it is incorporated into the ongoing treatment of their substance use and mental disorders [10,27]. Closer monitoring during smoking cessation treatment, including the individual psychiatric consultations performed in the present study, could be beneficial because of the specific role that tobacco may play in this subpopulation, such as tobacco self-medication. It may be that the close monitoring performed by these treatment staff reduced the gaps in smoking cessation rates suggested by other studies [4], because clinic staff were able to monitor the effects of tobacco 
Table 4

Multivariate regression model for 30-day continuous abstinence among smokers $(n=696)$ treated in CAPS-AD São Caetano do Sul. 2007-2013.

\begin{tabular}{lllll}
\hline & $\mathrm{OR}$ & $\mathrm{z}$ & $95 \% \mathrm{CI}$ & $\mathrm{p}$ \\
\hline Mental/Substance Use Disorder & 0.98 & -0.07 & $0.69-1.39$ & 0.945 \\
Time of smoking & 1.33 & 3.4 & $1.13-1.58$ & 0.001 \\
Systemic Arterial Hypertension & 1.27 & 1.29 & $0.88-1.82$ & 0.197 \\
Heaviness of Smoking Index & 0.71 & -4.13 & $0.61-0.84$ & $<0.001$ \\
Nicotine patch & 2.96 & 2.16 & $1.10-7.95$ & 0.031 \\
Buproprion & 1.45 & 2.17 & $1.03-2.03$ & 0.030 \\
Gender & 0.90 & -0.57 & $0.63-1.28$ & 0.571 \\
\hline
\end{tabular}

Model fit index: Log likelihood $=-422.2$ Pseudo 0.0440 .

treatment and adjust tobacco and psychiatric medications to avoid adverse effects and optimize outcomes. This may have been especially useful in Brazil, which still uses typical antipsychotics among a relatively high proportion of patients compared to many countries.

A combination of pharmacotherapy and group-based therapy (following cognitive-behavioral principles) is the most effective $[3,10,15,27]$. Health facilities that provide close monitoring of smokers and support the combination of behavioral and pharmacological treatment have success rates for MSUD patients similar to the success of treatments administered to the general population $[3,15]$. However, like many prior studies, we did not verify selfreported outcomes and only followed patients for a short while. These findings must therefore be viewed with caution.

According to a recent Cochrane review, all types of NRT (gum, patch patched, nasal spray, tablets/lozenges) increase smoking cessation rates by $50-70 \%$ [28]. Some groups such as pregnant women, young people and patients with psychiatric comorbidities may need more intensive psychotherapy, and be monitored for adverse or interactive pharmacological effects [29]. However, there are few studies that test tailored pharmacological regimens for smokers with MSUD.

Originally marketed as an atypical antidepressant, bupropion was discovered as a smoking cessation agent during a psychiatric trial [30]. It increased smoking cessation rates in patients with depression and rapidly became the non-nicotine drug of choice for the treatment of tobacco dependence [30]. Smokers treated with bupropion are less irritable, better able to concentrate, have less desire to smoke and experience less negative affect [31,32]. These benefits may be even more salient when treating patients with MSUD as they may have several underlying symptoms that mirror or magnify symptoms of withdrawal during cessation. A recent systematic review and meta-analysis suggested that bupropion is effective and tolerable for smoking cessation in adults with serious mental illnesses [33]. There are differences in nicotine-related effects and in the type of reinforcement produced when bupropion and NRT are compared. This generates conflicting data about the neural mechanisms used by bupropion in aiding smoking cessation, and may explain the beneficial clinical effect of the association between bupropion and NRT in smoking cessation treatment [34].

Despite the availability of treatment for smokers through the Brazilian National Cancer Institute (INCA), and the high prevalence of smoking among people with MSUD, this population has received little help to quit smoking in Brazil. These low levels of treatment are potentially due to a lack of information and false perceptions among health professionals regarding the treatment of smoking in this group of patients [35]. Data from this study, accompanied by information on the benefits of quitting and effective treatment methods, may help professionals embrace tobacco treatment as a life-affirming adjunct to psychiatric care.

Psychosocial units for alcohol and drugs (CAPS-AD) are specifically designed for individuals with substance use disorders
[3], and are largely integrated with the regular CAPS units (designed for mental health disorders). These units (CAPS and CAPS-AD) have staff from a variety of professions [36], and are well positioned to quickly adopt and effectively implement tobacco dependence treatment [15].

\subsection{Limitations}

The main limitations of this study are related to the fact that abstinence was assessed based on the patients' reports. Tests of salivary cotinine and carbon monoxide would have helped determine the absolute effects of treatment [37]. Because tobacco relapse is common [38], longer follow up would have better estimated the long-term effects of this protocol. Unfortunately, just a few patients were prescribed nortriptyline, which may have generated a type-one error in terms of evaluating its effectiveness relative to the more popular bupropion and NRT. Varenicline, an important medication in the treatment of tobacco addiction [9], was not used in this study because it was not available through the National Smoking Cessation Program. This study did not include a diagnostic tool to assess the presence and the type of MSUD disorders, which limits our ability to pinpoint treatment effects among patients with different diagnoses.

The 6-week protocol used in the present study is short. Tobacco use, like other drug disorders, is a chronic relapsing condition and it is likely that longer treatment could have resulted in higher quit rates. Furthermore, this study described the outcomes of smoking cessation intervention using standard practices in Brazil, including group therapy sessions combined with cessation medications. There are, however, no written manual or protocols for these sessions. As such, it will be difficult for other units to replicate the treatment approach.

\subsection{Conclusion}

In summary, our findings both confirm and add to those of previous studies in the general population. Factors predictive of smoking cessation in the general population also predicted success among individuals with MSUD. This was an observational real-life study, which rends it both strengths and weaknesses. Unfortunately, there is a lack of studies like this. The main contribution of the present study is the finding of similarities between individuals with and without MSUD regarding smoking cessation treatment, when they are treated 'together'. This is important, because individuals with MSUD suffer a lot from stigma and one of the outcomes of this is the lack of smoking cessation treatment offering, even in mental health/addiction care units. The inclusion of both kind of patients in the same treatment protocol could increase the success rates among individuals with MSUD, and ultimately, diminishing stigma attached to individuals with MSUD. To improve the length and quality of life among people with MSUD, units that specialize in the treatment of this vulnerable population, such as CAPS in Brazil, should be encouraged to proactively treat tobacco dependence.

\section{Disclosure of interest}

Dr. Castaldelli-Maia has been awarded with a Independent Grant for Learning and Change (IGLC) from Pfizer, managed by Global Bridges (Healthcare Alliance for Tobacco Dependence Treatment) hosted at Mayo Clinic, to support free smoking cessation treatment training in addiction/mental health care (CAPS) units in Brazil (grant IGLC 13513957) and Portugal (grant IGLC 25629313). Dr. Andrade is Executive President of Center for Information on Health and Alcohol (CISA), which had no funding relationship with this project. All other authors declare no conflict of interest. 


\section{References}

[1] World Health Organization. WHO Report on the Global Tobacco Epidemic: Warning About the Dangers of Tobacco. Geneva: World Health Organization; 2011.

[2] Anthenelli RM, Benowitz NL, West R, St Aubin L, McRae T, Lawrence D, Ascher J, Russ C, Krishen A, Evins AE. Neuropsychiatric safety and efficacy of varenicline, bupropion, and nicotine patch in smokers with and without psychiatric disorders (EAGLES): a double-blind, randomised, placebo-controlled clinical trial. Lancet 2016;387:2507-20.

[3] Castaldelli-Maia JM, Loreto AR, Carvalho CFC, Frallonardo FP, de Andrade AG. Retention predictors of a smoking treatment provided by a public psychosocial unit in Brazil. Int Rev Psychiatry 2014;26:515-23.

[4] Evins AE, Cather C, Laffer A. Treatment of tobacco use disorders in smokers with serious mental illness: toward clinical best practices. Harv Rev Psychiatry 2015;23:90-8.

[5] Ziedonis D, Hitsman B, Beckham JC, et al. Tobacco use and cessation in psychiatric disorders: national Institute of Mental Health report. Nicotine Tob Res 2008; 10:1691-715.

[6] Colton CW, Manderscheid RW. Congruencies in increased mortality rates, years of potential life lost, and causes of death among public mental health clients in eight states. Prev Chronic Dis 2006;3:A42.

[7] Lasser K, Boyd JW, Woolhandler S, Himmelstein DU, McCormick D, Bor DH. Smoking and mental illness: a population-based prevalence study. JAMA 2000;284:2606-10.

[8] Cahill K, Stevens S, Perera R, Lancaster T. Pharmacological interventions for smoking cessation: an overview and network meta-analysis. Cochrane Database Syst Rev 2013;5:CD009329.

[9] Stead LF, Koilpillai P, Fanshawe TR, Lancaster T. Combined pharmacotherapy and behavioural interventions for smoking cessation. Cochrane Database Syst Rev 2016;3:CD008286.

[10] Hitsman B, Moss TG, Montoya ID, George TP. Treatment of tobacco dependence in mental health and addiction disorders. Can J Psychiatry 2009;54:368-78.

[11] McFall M, Saxon AJ, Malte CA, et al. Integrating tobacco cessation into mental health care for posttraumatic stress disorder: a randomized controlled trial. JAMA 2010;304:2485-93.

[12] Prochaska JJ, Hall SM, Tsoh JY, et al. Treating tobacco dependence in clinically depressed smokers: effect of smoking cessation on mental health functioning. Am J Public Health 2008;98:446-8.

[13] Prochaska JJ. Integrating tobacco treatment into mental health settings. JAMA 2010;304:2534-5.

[14] Castaldelli-Maia JM, Ventriglio A. The impact of Basaglia on Brazilian psychiatry. Int J Soc Psychiatry 2016;62:411-4.

[15] Castaldelli-Maia JM, Carvalho CF, Armentano F, et al. Outcome predictors of smoking cessation treatment provided by an addiction care unit between 2007 and 2010. Rev Bras Psiquiatr 2013;35:338-46.

[16] Miranda L, Campos RTO. Análise do trabalho de referência em Centros de Atenção Psicossocial. Rev Saude Publica 2008;42:907-13.

[17] Chengappa KN, Perkins KA, Brar JS, et al. Varenicline for smoking cessation in bipolar disorder: a randomized, double-blind, placebo-controlled study. J Clin Psychiatry 2014;75:765-72.

[18] Willenbring ML, Kivlahan D, Kenny M, Grillo M, Hagedorn H, Postier A. Beliefs about evidence-based practices in addiction treatment: a survey of Veterans Administration Program leaders. J Subst Abuse Treat 2004;26:79-85.
[19] Schroeder SA, Morris CD. Confronting a neglected epidemic: tobacco cessation for persons with mental illnesses and substance abuse problems. Annu Rev Public Health 2010;31:297-314.

[20] American Legacy Foundation. A Hidden Epidemic: Tobacco Use and Mental Illness. Washington, D.C: American Legacy Foundation; 2011.

[21] Loreto AR, Carvalho CFC, Frallonardo FP, Ismael F, Andrade AG, Castaldelli-Maia JM. Smoking cessation treatment for patients with mental disorders using CBT and combined pharmacotherapy. J Dual Diagn 2017;13:238-46.

[22] Etter JF, Duc TV, Perneger TV. Validity of the Fagerström test for nicotine dependence and of the Heaviness of Smoking Index among relatively light smokers. Addiction 1999;94:269-81.

[23] Walker N, Howe C, Glover M, et al. Cytisine versus nicotine for smoking cessation. N Engl J Med 2014;371:2353-62.

[24] Capron DW, Allan NP, Norr AM, Zvolensky MJ, Schmidt NB. The effect of successful and unsuccessful smoking cessation on short-term anxiety, depression, and suicidality. Addict Behav 2014;39:782-8.

[25] Pachas GN, Cather C, Pratt SA, et al. Varenicline for smoking cessation in schizophrenia: safety and effectiveness in a 12-week, open-label trial. J Dual Diagn 2012;8:117-25.

[26] Evins AE, Cather C, Deckersbach T, et al. A double-blind placebo-controlled trial of bupropion sustained-release for smoking cessation in schizophrenia. J Clin Psychopharmacol 2005;25:218-25.

[27] Morisano D, Bacher I, Audrain-McGovern J, George TP. Mechanisms underlying the comorbidity tobacco use in mental health and addiction disorders. Can Psychiatry 2009;54:356-67.

[28] Stead LF, Perera R, Bullen C, et al. Nicotine replacement therapy for smoking cessation. Cochrane Database Syst Rev 2012;11:CD000146.

[29] Batra A, Friederich HM, LutzU.Treatment of tobacco dependence: a responsability of the psychiatry and the addiction medicine. Nervenarzt 2009;80:1022-9.

[30] Raw M, Regan S, Rigotti NA, McNeill A. A survey of tobacco dependence treatment guidelines in 31 countries. Addiction 2009;104:1243-50.

[31] Shiffman S, Johnston JA, Khayrallah M, et al. The effect of bupropion on nicotine craving and withdrawal. Psychopharmacology 2000;148:33-40.

[32] Strong DR, Kahler CW, Leventhal AM, et al. Impact of bupropion and cognitivebehavioral treatment for depression on positive affect, negative affect, and urges to smoke during cessation treatment. Nicotine Tob Res 2009;11:1142-53.

[33] Roberts E, Eden Evins A, McNeill A, Robson D. Efficacy and tolerability of pharmacotherapy for smoking cessation in adults with serious mental illness: a systematic review and network meta-analysis. Addiction 2016;111:599-612.

[34] Evins AE, Cather C, Culhane MA, et al. A 12-week double-blind, placebocontrolled study of bupropion sr added to high-dose dual nicotine replacement therapy for smoking cessation or reduction in schizophrenia. J Clin Psychopharmacol 2007;27:380-6.

[35] Fagerström K, Aubin HJ. Management of smoking cessation in patients with psychiatric disorders. Curr Med Res Opin 2009;25:511-8.

[36] Mielke FB, Kantorski LP, Jardim VM, Olschowsky A, Machado MS. Mental care delivered in psychosocial care centers (CAPS) from the viewpoint of the professionals. Cien Saude Colet 2009;14:159-64.

[37] Aveyard P, Bauld L. Incentives for promoting smoking cessation: what we still do not know. Cochrane Database Syst Rev 2011;8:ED000027.

[38] Taylor M, Leonardi-Bee J, Agboola S, McNeill A, Coleman T. Cost effectiveness of interventions to reduce relapse to smoking following smoking cessation. Addiction 2011;106:1819-26. 